\title{
Herding to a side of Order Book Balance
}

\author{
Hokky Situngkir*(a,c), Yohanes Surya ${ }^{(b)}$ \\ (a) Dept. Comp. Soc., Bandung Fe Institute, Indonesia \\ (b) Surya Research International, Jakarta, Indonesia
}

\begin{abstract}
In the growing econophysics, it is quite rare that the presented analyses approach the interesting properties of order book. However, a lot of data are available in the order book, and analysis on this object will bring us to further understanding of the market. We analyze some order book of stocks traded in Jakarta Stock Exchange and see interesting properties there by looking at the balance of the order book. We also build a model based on the work of Chiarella and Iori [1] with some modifications to explain the herding pattern of traders through the order book.
\end{abstract}

\section{Keywords:}

econophysics, order book imbalance, Indonesian stock market.

* corresponding author, mail: hs@compsoc.bandungfe.net 


\section{Introduction}

There have been a lot of variations of trading system in stock markets all over the world regarding the order submissions, the matching processes, including the way investors/traders access the order book. Order book shows when, who, and how the traders submit their buy/sell orders including the size and the price level of the orders. Most analyses in econophysics are trying to understand the price formation just by looking at the price dynamics while there are also a lot of interests to see microstructure of the pre-matching process of buy/sell orders by approaching the queueing orders in the order book.

An interesting and unique thing we found in most stocks traded in Jakarta Stock Exchange is that in most cases, a lot of submitted orders are placed too far from price level of the best orders (best-bid and best-ask) that showed an imbalance in the order book. Technically speaking, the orders are placed too far in the uncompetitive region which is almost impossible to be matched during the trading day [3]. However, an effect of this submissions in return can influence other traders observing the order book and bring some kind of psychological image about the imbalance [4]. We know from the principle of economics that excess demand brings about the rise of price and excess supply brings the downward movement.

From here, we can clearly see the fact that balance of supply and demand in the order book play an important role for traders' decision making, of course, together with the emerging (historical) price dynamics. In the perspective of macro-micro description level of a stock market, we can draw the diagram as showed by figure 1 . In other words, the herding behavior can occur from the information acquired from the order book as well as the price dynamics.

Interestingly, we can see from the figure that interaction of trading agents emerges the statistical properties of the order book as well as the price formation in the macro layer. In return, the price formation and the dynamics of the order book will also be put into account by traders making their decision to be submitted.

The herding behavior is a microstructural situation emerging the volatility clustering and the leptokurtic of the distribution of return [2]. Focusing to the traders' observation to the order book, we can obviously say that the psychological image of the order book imbalance can also lead to the herding behavior. As imbalance of the order book tend bigger to the excessive size of demand the price will potentially move upwards, and vice 


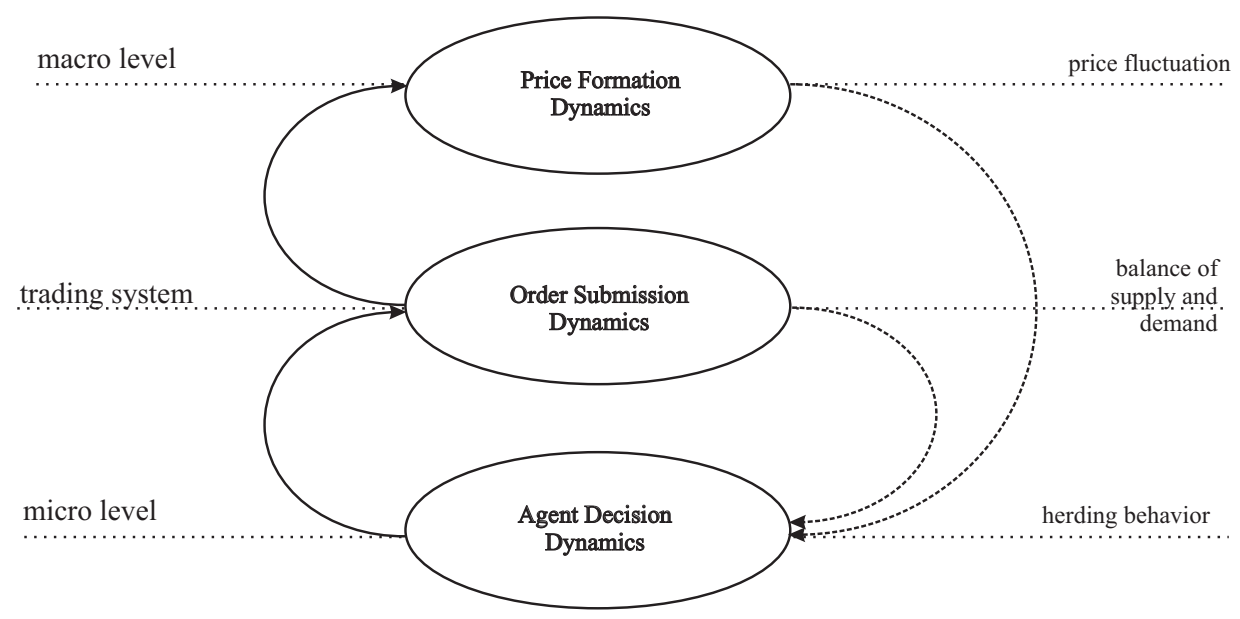

Figure 1: The market microstructure: the agent's decision, the order submission, and the price formation

versa.

The paper adapted the visualization introduced in [4] to see the balance of the empirical order book of some stocks traded in Jakarta Stock Exchange. Furthermore, we will do the simulation showing the herding of order submission as the imbalance between supply and demand grows.

\section{The Balance of Order Book}

The analysis of order book balance is related to the the size and price level of the submitted orders. The closer submitted orders to the best order, the more competitive it is, for the probability to be matched is obviously higher. In this paper, we visualize the balance of the buy/sell orders by denoting the order size as the function of price level, $Q(\Delta x)$. The buy $(\beta)$ and sell $(\alpha)$ orders in the order book can simply be written as,

$$
\ldots \beta_{3} \leq \beta_{2} \leq \beta_{1} \leq \alpha_{1} \leq \alpha_{2} \leq \alpha_{3} \cdots
$$

Here, $\beta_{1}$ denotes the best (i.e.: highest) bid price, $\beta_{2}$ the second best, and so on, as well as $\alpha_{1}$ denotes the best (i.e.: lowest) ask price, $\alpha_{2}$ the second best, and so on. To ease our visualization, each price level is assigned into positive integer (sell orders) and negative integer (buy orders). We draw the balance order book as showed in figure 2 and 3. 


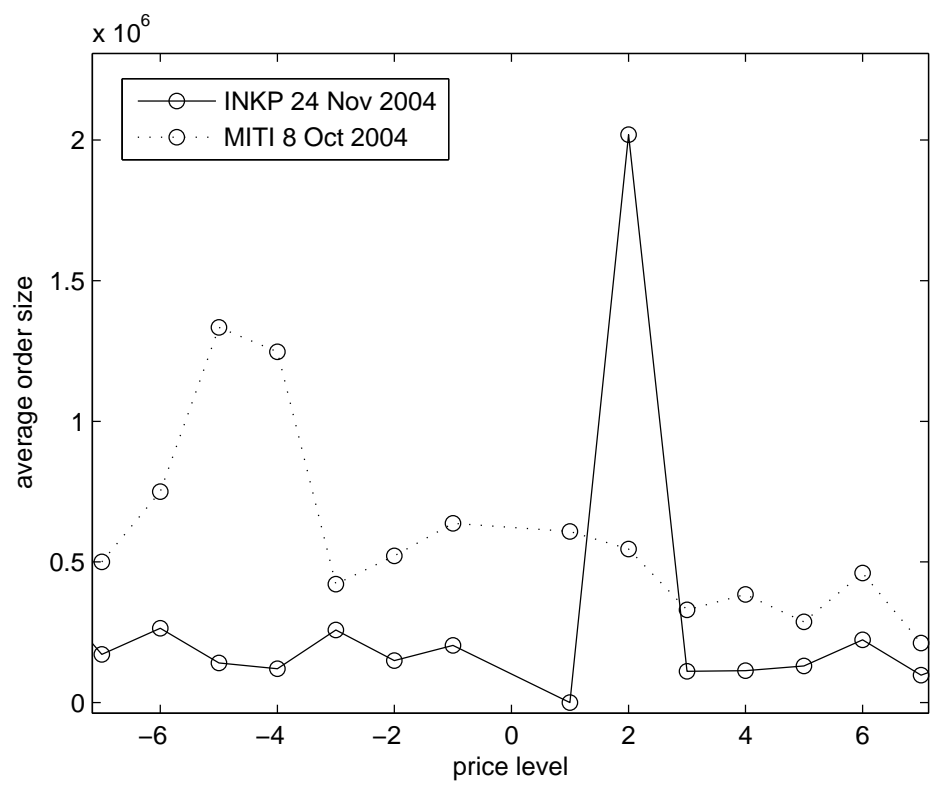

Figure 2: The visualization of balance order book of stocks INKP and MITI traded in JSX

In the visualizations, we can see that the vertical axis representing the average of order size and the horizontal one the price levels numbered by the index of the level. -1 is the best bid, -2 the second best bid, and so on, while 1 is the best ask, 2 the second best ask, and so on.

It is an obvious difference of what is visualized in figure 2 and in figure 3 . The latest shows how orders are accumulated in the twenty five best bid for BRPT stock while for CTRA stock they are piled up in eighteen and twenty best ask. Surely in some way this could influence other traders observing the order book. In BPRT stock trading there is a psychological image that there is a big push to rise the current price while in the CTRA it is some kind of a huge push downward. This is somewhat a frequent experience in some Indonesian stocks related to their liquidity (see [8] and [9]). However, it also becomes such a "trading strategy" for some illiquid stocks.

\section{Simple Model of Market Microstructure}

We adapted the model in [1] to understand the micro properties of the herding behavior through the imbalance of the order book. Different with the previous work [7] and [9], 


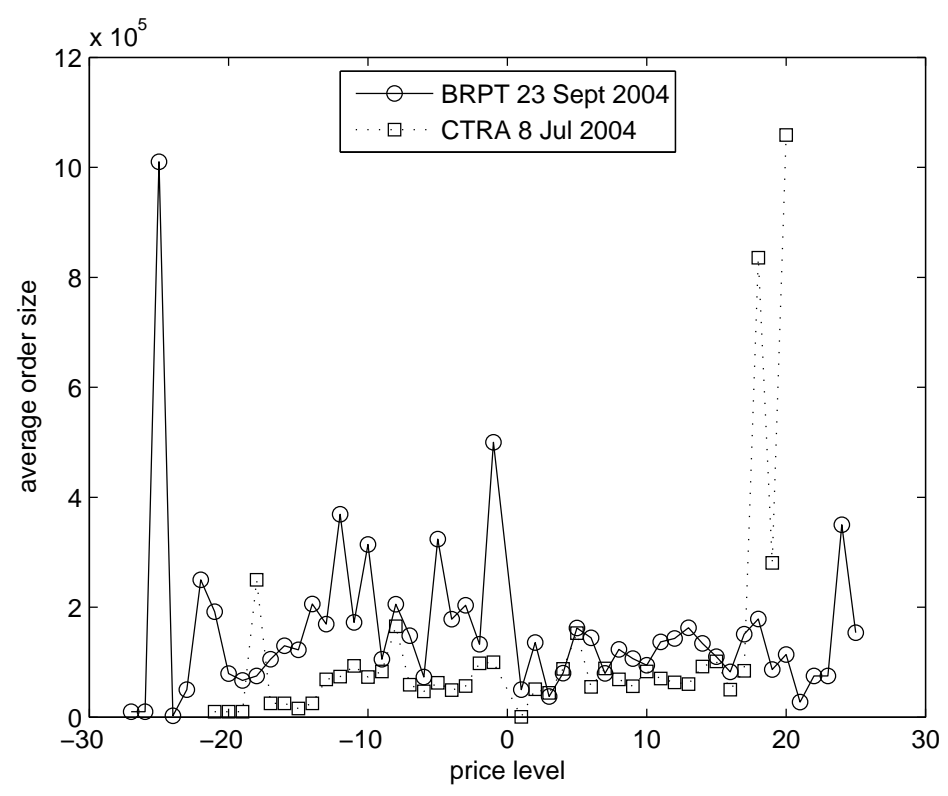

Figure 3: Balance order book of BRPT and CTRA showing the herding of orders piled up in far levels from the competitive area.

we do not categorize the strategy of the agents in a strict way. An agent simply has tendencies of being a chartist, fundamentalist, or noise trader in such a way of calculating their expected return by using,

$$
\hat{r}_{t, t+\tau}^{i}=\frac{1}{s_{1}^{i}+\left|s_{2}^{i}\right|+n^{i}}\left[s_{1}^{i} \frac{1}{\tau_{f}} \frac{\left(x^{f}-x_{t}\right)}{x_{t}}+s_{2}^{i} \bar{r}_{\tau^{i}}+n^{i} \epsilon_{t}\right]
$$

where

$$
\begin{array}{lll}
x_{f} & : \text { agent's fundamental price } \\
x_{t} & : \text { price at } t \\
\hat{r}_{t, t+\tau}^{i} & : \text { average return in the time interval } \tau \text { calculated by agent } i \\
s_{1}^{i} & : \text { agent's weight as fundamentalist, } s_{1}^{i} \sim\left|N\left(0, \sigma_{1}\right)\right| \\
s_{2}^{i} & : \text { agent's weight as chartist, } s_{2}^{i} \sim N\left(0, \sigma_{2}\right) \\
n^{i} & : \text { noise component of agent } i, n^{i} \sim\left|N\left(0, \sigma_{n}\right)\right| \\
\epsilon^{t} & : \text { global noise component changing over time, } \epsilon^{t} \sim N\left(0, \sigma_{\epsilon}\right) \\
\tau_{f} & : \text { fundamentalist's horizon, } \\
& & \text { the length of time in which fundamentalist expecting price move to } x_{f}
\end{array}
$$

Concerning each agent's time horizon, we understand that fundamentalists have much 


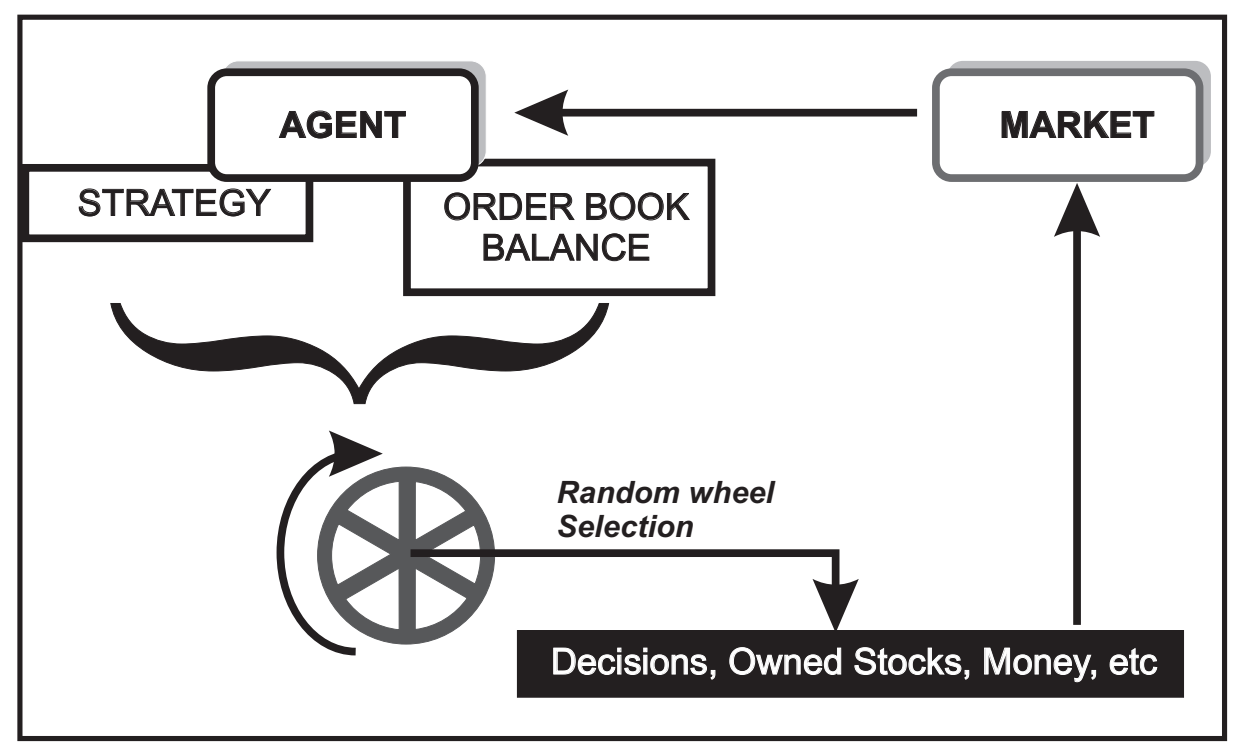

Figure 4: The simulation of our order book dynamics

longer time horizon relative to the chartists. Thus, each agent's horizon is,

$$
\tau_{i}=\left[\tau^{c} \frac{1+s_{1}^{i}}{\left|1+s_{2}^{i}\right|}\right]
$$

where $\tau^{c}$ is the referential time horizon.

The average return $\bar{r}_{L^{i}}$ in the interval of $\tau^{c}$ is simply defined as

$$
\bar{r}_{L^{i}}=\frac{1}{\tau^{i}} \sum_{j=1}^{\tau^{i}} \log \frac{x_{t-j}}{x_{t-j-1}}
$$

One should note that what we want to figure out here is the per tick dynamics in the stock market. Thus, each round (iteration) represents one tick trading. Technically, in every round, each agent has certain probability $(\rho)$ to place an order $\left(o_{t}^{i}\right)$ regarding her expectation of the future value of the stock. Obviously, if she expects that price will go up, she will submit a buy order, and vice versa.

For the sake of simplicity, we treat all order as limit order, since market order (order to be matched with best quote) can also be regarded as limit order whose level is the best quote. If we assign a buy order as 1 and sell as -1 , hence

$$
o_{t}^{i}= \begin{cases}1, & \hat{x}_{t+\tau^{i}}^{i}>x_{t} \\ -1, & \hat{x}_{t+\tau^{i}}^{i}<x_{t}\end{cases}
$$


where

$$
\hat{x}_{t+\tau^{i}}^{i}=x_{t} \exp \left(\hat{r}_{t, t+\tau}^{i} \tau^{i}\right)
$$

In our model, there is also probability $\left(\rho^{*}\right)$ that an agent can be influenced by the imbalance of the order book since she can observe the real time order book. Thus, agents can also submit their orders not merely because of their trading strategy (i.e.: fundamentalist, chartist, and noise) but also the result of their evaluation of the order book. If the

$$
o_{t}^{i i}= \begin{cases}1, & Q_{b i d}>Q_{a s k} \\ -1, & Q_{b i d}<Q_{a s k}\end{cases}
$$

where $Q_{b i d}$ and $Q_{a s k}$ are the total quantity of buy and sell quotes respectively. Thus, each agent will have two opportunities to submit order in each round: based on her strategy (with probability $\rho$ ) and based on her observation of all the quotes (with probability $\rho^{*}$ ).

For each of them, the price level of a buy $\left(b_{t}^{i}\right)$ and sell $\left(a_{t}^{i}\right)$ order

$$
\begin{aligned}
& b_{t}^{i}=\hat{x}_{t+\tau^{i}}^{i}\left(1-k^{i}\right) \\
& a_{t}^{i}=\hat{x}_{t+\tau^{i}}^{i} /\left(1-k^{i}\right)
\end{aligned}
$$

with $k$ is a random number uniformly distributed in the interval $(0,1)$. Certainly, an agent must evaluate her financial condition before submitting any orders. She will never be able to place a buy order if she lacks of money and can not submit a sell order if she does not have anything to sell. The way agents trade, interact, place orders, are showed in figure 4 .

Apparently, the order submission from agent will never directly be mapped onto real numbers since price is a discrete variable with $\delta$ as the smallest size. Furthermore, the agent also submit the size of buy/sell order. Here we use the assumption of neutral risk agents. Thus, the size of the order is simply a random demand function,

$$
\begin{gathered}
\left.v^{i}\right|_{o_{t}^{i}=1}=\theta \frac{U_{t}^{i}}{b_{t}^{i}} \\
\left.v^{i}\right|_{o_{t}^{i}=-1}=\theta M_{t}^{i}
\end{gathered}
$$



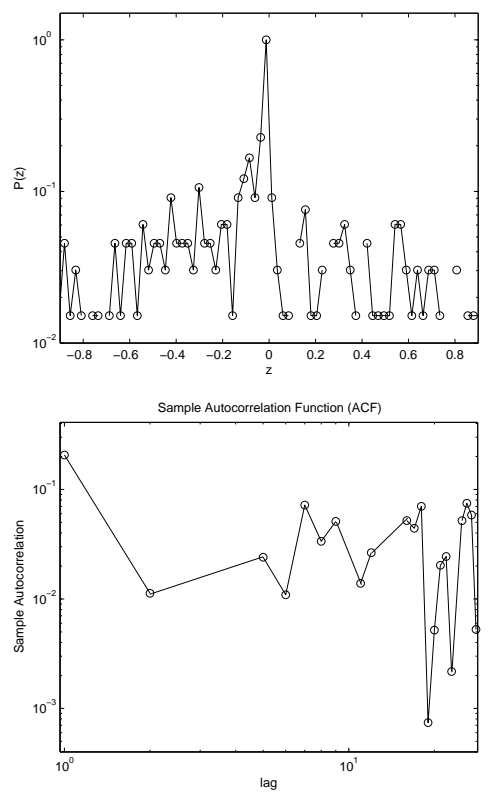

Figure 5: The leptokurtic distribution of return (top) and the autocorrelation of the absolute price return (bottom) yielded from the simulation.

where $U_{t}^{i}$ and $M_{t}^{i}$ are the amount of money to be invested and amount of stocks owned by agent $i$ at time $t$ respectively, while $\theta$ is a uniformly distributed random number.

For the price formation, in each round we use middle value of the quoted sell $\left(a_{t}^{q}\right)$ and buy order $\left(a_{t}^{q}\right)$.

$$
x_{t}=\frac{a_{t}^{q}+b_{t}^{q}}{2}
$$

We discuss our simulation result in the next section, that we find that the accumulation of orders far from the competitive price level can occur as an effect of unique herding behavior through the imbalance of the order book.

\section{Discussions}

The result of the simulation is showed in figure 5. It shows the leptokurtic distribution of return and the autocorrelation of absolute price return. In advance, our focus in the simulation is to see the imbalance order book in rounds of our simulation. We do this by comparing the balance order book visualization with and without the direct access of agents to the order book. 

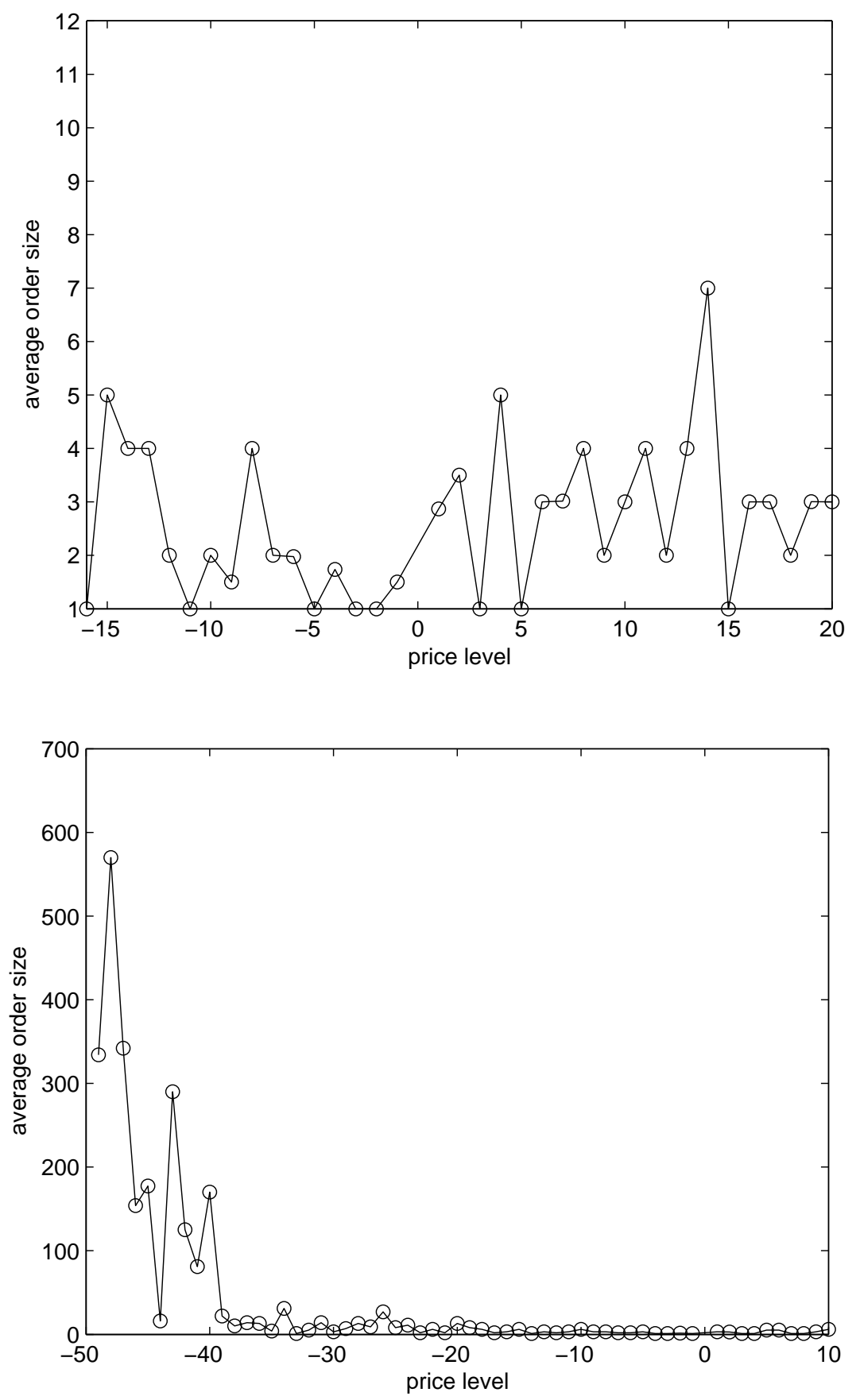

Figure 6: The order book balance after rounds of simulations: simulation without agent's access to the order book $(t o p)$ and with agent's opportunity to evaluate the balance of order book (bottom). 
The order book balance as yielded in our simulation can be seen in figure 6 . As we have assumed before, we get different result between the simulation with and without agent's ability to access the order book. Since in our simulation an agent has opportunity to access the order book, she can manage her order placement by evaluating in short term the balance of the order book. Thus, a highly fundamental agent, for example, can possibly place a buy order to fulfil her portfolio but as she observed the imbalance of the order book to the ask-side, she also submit a buy order with smaller quantity. We assume the frequent imbalance of the order book in some stocks traded in JSX caused by such strategies of trading.

In return, there is also a possible strategy for a highly capitalized agent to try to move the price as she placed a big order not in a competitive price level in order to influence other traders. However, this can only be done in not-so-liquid stocks since in a more liquid stock, the dynamics of the order book and price fluctuations are relatively so fast and hard to observe even in a very short horizon.

\section{Concluding Remarks}

We have shown the frequent imbalance in the order book of some stocks traded in JSX and how this fact related to the liquidity of the stocks. The imbalance in the order book can give psychological effect to traders and influence their trading strategies. To see this clearly we do computer simulation by comparing the simulation with and without the ability of agents evaluating the dynamics of the order book.

Relating this fact to the liquidity of the stocks, it could be a kind of additional strategy for agents on placement of their orders for short term (e.g.: intra-day) trading in the illiquid stocks whose price is rarely moved. However, this can be harmful for the stocks since the respective stock price will be easily driven by bunch of investors. A research for this has been conducted in JSX and reported in [10].

\section{Acknowledgement}

Both authors thank the Jakarta Stock Exchange (JSX) and Surya Research International (SRI) for financial support in the period of the research. We also thank JSX Research Division especially Kandi Sofia Dahlan and Vitri Herma Susanti for discussions about order book analysis. HS thank BFI researchers for input in the earlier draft of this paper and Deni Khanafiah for data processing. 


\section{References}

[1] Chiarella, C. and Iori, G. (2004). "The Impact of Heterogeneous Trading Rules on the Limit Order Book and Order Flows." Research Paper Series 152, Quantitative Finance Research Centre, University of Technology, Sydney.

[2] Cont, R. and Bouchaud, J. (2000). "Herd Behavior and Aggregate Fluctuations in Financial Markets. Macroeconomic Dynamics 4:170-96

[3] Luckock, H. (2003). "A Steady-state model of the Continuous Double Action". Quantitative Finance 3:385-404.

[4] Maslov, S. and Mills, M. (2001). "Price Fluctuation from the Order Book Perspective - empirical facts and a simple model". Physica A 299:234-246.

[5] Smith, E ., Farmer, J. D., Gillemot, L., dan Krishnamurthy, S. (2003). "Statistical Theory of the Continuous Double Action". Quantitative Finance 3: 481-514. Institute of Physics Publishing.

[6] Situngkir, H. and Surya, Y. (2003). "Stylized Statistical Facts of Indonesian Financial Data: Empirical Study of Several Stock Indexes in Indonesia". Prosiding Simposium Himpunan Fisika XX. Pre-print: arxiv: cond-mat/0403465.

[7] Situngkir, H. and Surya, Y. (2004). Statistical Facts of Artificial Stock Market. Preprint: arXiv:cond-mat/0408358.

[8] Situngkir, H. and Surya, Y. (2005a). On Stock Market Dynamics through Ultrametricity of Minimum Spanning Tree. Working Paper WPH2005 Bandung Fe Institute. URL: http://www.bandungfe.net/wp2005/2005h.pdf

[9] Situngkir, H., Hariadi, Y. and Surya, Y. (2005b). Membandingkan Continuous dan Continuous-Interval Trading System Dalam Perspektif Likuiditas: Simulasi Mikrostruktur. Research report in the cooperation between SRI and BEJ in the period February-April 2005.

[10] Situngkir, H., Hariadi, Y. and Surya, Y. (2005c). Pola Transaksi dalam Kaitannya dengan Herding Behavior. Research report in the cooperation between SRI and BEJ in the period June-December 2005. 